\title{
The Design of Defect Detection Algorithm for the Energy-saving Lamp Tungsten
}

\author{
Gu Qinlong ${ }^{1, a}$, Chen Defu ${ }^{1, b}$, Shen Qiang ${ }^{1}$ and Yao Minghai ${ }^{1, c^{*}}$ \\ ${ }^{1}$ College of Information Engineering,Zhejiang University of Technology, Hangzhou,China \\ agql@zjut.edu.cn, bdefuchen@zjut.edu.cn, cymh@zjut.edu.cn
}

Keywords: energy-saving lamp, defect check, image recognition, ROI area

Abstract. In the energy-saving lamp production, there may be some defects such as no lamp post, too long tail, excess or not enough powder coating. The paper proposes the defect detection algorithm based on tungsten defect features with machine vision. It contains obtaining of tungsten area, filtering, marking out of image coordinate, feature extraction and tungsten image defect detection. The work is completed in Embedded system with TMS320DM6467 processor and Davinci software framework. The experiment shows the algorithm is effective.

\section{Introduction}

The major application of machine vision ${ }^{[1][2]}$ is to check deficient product. The test method is contactless. The field of application of machine vision covers defect check and classification of metal parts, fabric defect detection, surface defect check of car body, glass defect detection and so on ${ }^{[3]}$. Because machine vision depends on specific types of product and defect, it should apply with different recognition methods according to specific application. In the production process of energy-saving lamp, we need to apply a layer of powder slurry on tungsten evenly and completely, that is tungsten powder coating ${ }^{[4]}$. For the reason coating process errors and the defect of energy-saving lamp post, there may be some flaws such as no lamp post, too longer tail, more powder coating and powder coating incompletely in this process. In production of enterprises at present time, the quality of tungsten powder coating is monitored by manpower. If technology of machine vision is applied, the process of tungsten powder coating can be manufactured and monitored automatically, that can save manpower, enhance the quality of energy-saving lamp and production efficiency.

The paper puts forward an algorithm to realize defect check of the energy-saving lamp post, tail and filament. The flow chart of the algorithm is shown in Fig. 1. After image inversion, de-nosing, filtering $^{[5]}$, and image segmentation preprocess, energy-saving lamp post, tail and filament check is carried out.

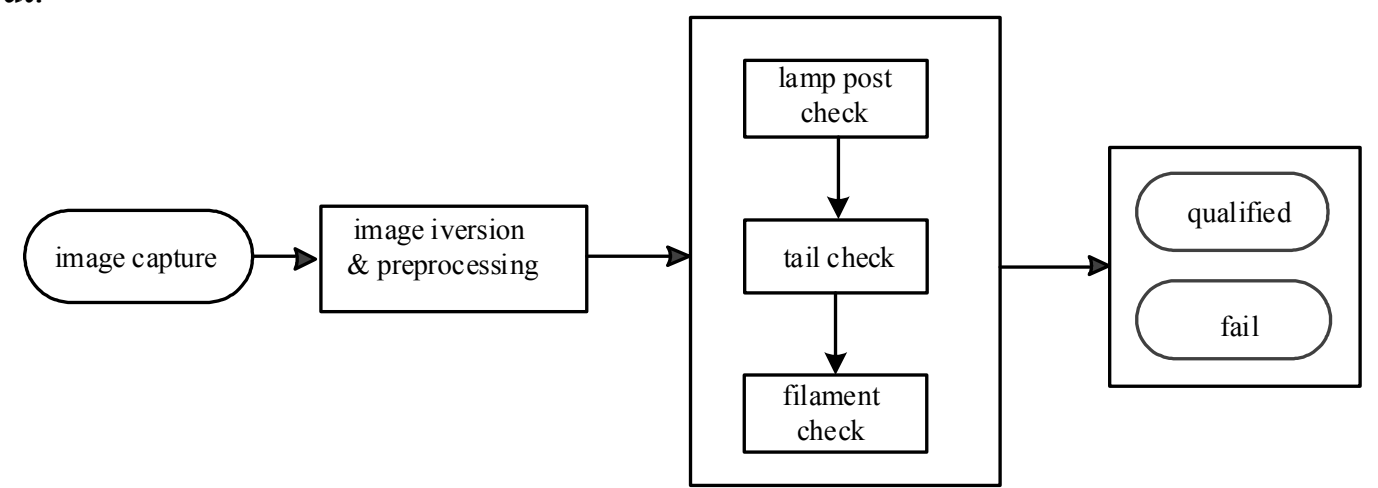

Fig. 1 Flow chart of the algorithm for defect check of the energy-saving lamp tungsten

The input of the algorithm is RGB format image. We should preprocess the image to enhance the quality of input image and save process time. We firstly extract ROI(Region of interest) area, then translate RGB image into grayscale and carry out binary conversion. ROI is the abbreviation of specific area that need to deal with, that can be rectangle, circle, eclipse and irregular polygon ${ }^{[6]}$. In this paper we extract rectangle ROI area to check energy-saving lamp defect. 
With the development of semiconductor technology, it is possible for vision applications in embedded systems. For the reason that light weight, small size, low power consumption, high reliability and high resource utilization, it is the mainstream of development in the future that check deficient product based on machine vision in embedded solution. The work in this paper is carried out in development board configured with TMS320DM6467 processor $^{[7]}$ and in Davinci software framework $^{[8]}$. This paper is organized as follows. The lamp post, tail and filament defect check of energy-saving lamp is introduced in chapter 2, 3 and 4 separately. At the end of the paper, the experiment and conclusion is given.

\section{Energy-saving lamp post defect check}

There are some flaws that consist in energy-saving lamp post, such as no filament, one post and tail flaw, as shown in Fig. 2. The fundamental principle is to determine the coordinate figure of the energy-saving lamp post, that can be used to judge the defect type. The characteristic points of the energy-saving lamp post are marked out in Fig. 3. The algorithm of defect check for the energy-saving lamp post is illustrated in the following.
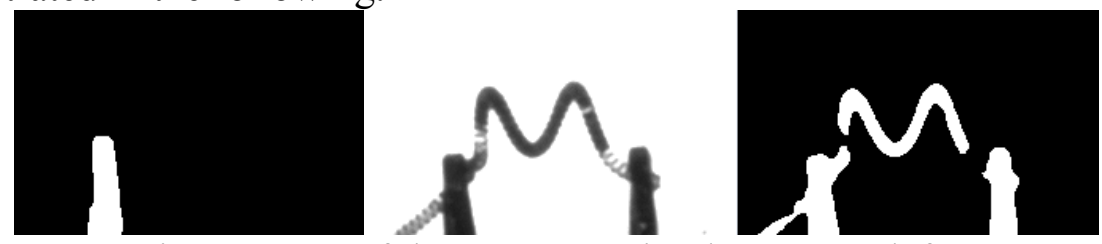

Fig. 2 Image of the energy-saving lamp post defect

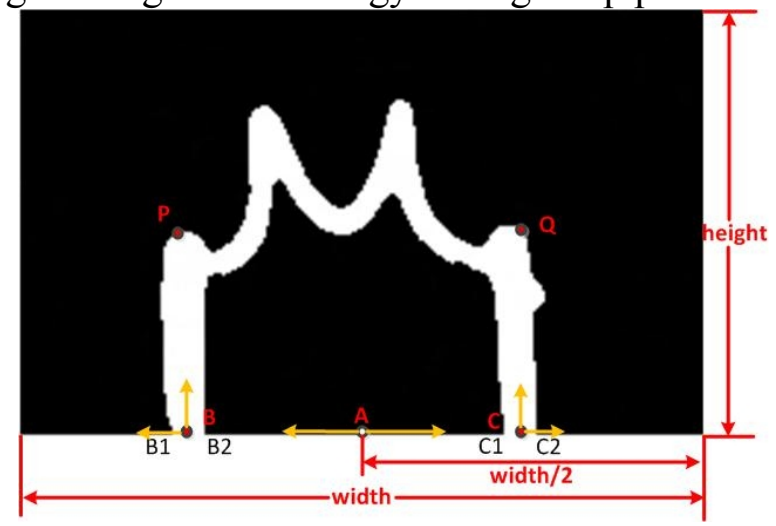

Fig. 3 Energy-saving lamp post marked with characteristic points

(1) get the mid-point coordinate of bottom boundary of figure 3 , that is $A(x, y)$.

(2) traverse to right boundary and left boundary of ROI-based image from point A.

For binary image,jump points should be traversed under normal circumstances. For the binary image of energy-saving lamp post, left jump points namely $B_{1}(x, y), B_{2}(x, y)$,right jump points namely $C_{1}(x, y), C_{2}(x, y)$. If there are just $\mathrm{B}$ or $\mathrm{C}$ points, the flaw can be judged as one post. If there are more points besides $\mathrm{B}$ and $\mathrm{C}$ points, that means tail wire is here. The corresponding energy-saving lamp is not good. If the energy-saving lamp has no post, ROI-based image should be blank and no points to be traversed.

For further check demand, the width and height of energy-saving lamp post should be given. Firstly, we figure out the central coordinate of right and left jump points. The central coordinate of the jump points of two sides is expressed as $B(x, y)$ and $C(x, y)$ respectively. The $\mathrm{x}$-axis and $\mathrm{y}$-axis of $\mathrm{B}$ point, named as $x_{B}$ and $y_{B}$, calculated in Eq. 1. The $\mathrm{x}$-axis and y-axis of C point, that is $x_{C}$ and $y_{C}$, calculated in Eq. 2. Then, the width of energy-saving lamp post can be gotten in Eq. 3.

$$
\left\{\begin{array}{l}
x_{B}=\frac{x_{B_{1}}+x_{B_{2}}}{2} \\
y_{B}=\text { height }
\end{array}\right.
$$




$$
\begin{aligned}
& \left\{\begin{array}{l}
x_{C}=\frac{x_{C_{1}}+x_{C_{2}}}{2} \\
y_{C}=\text { height }
\end{array}\right. \\
& d_{n}=\frac{x_{B_{2}}-x_{B_{1}}+x_{C_{2}}-x_{C_{1}}}{2}
\end{aligned}
$$

Now we derive the height of energy-saving lamp post. Assumed pixel coordinate of ROI-based image as $I(x, y)$, traverse to top boundary from B, the highest point, namely P point can be reached. Similarly, traverse to top boundary from $\mathrm{C}$, the highest point, namely $\mathrm{Q}$ point can be reached. Then, the height of energy-saving lamp post, namely $h_{P Q}$, can be gotten in Eq. 6 .

$$
\begin{aligned}
& \left\{\begin{array}{l}
x_{P}=x \\
y_{P}=\min \left(y_{i}\right)
\end{array}, I\left(x, y_{i}\right)-I\left(x, y_{i-1}\right) \neq 0, x \in\left(0, x_{B}\right), 0<i<\right.\text { height } \\
& \left\{\begin{array}{l}
x_{Q}=x \\
y_{Q}=\min \left(y_{i}\right)
\end{array}\right) I\left(x, y_{i}\right)-I\left(x, y_{i-1}\right) \neq 0, x \in\left(x_{C}, \text { width }\right), 0<i<\text { height } \\
& h_{P Q}=\frac{\text { height }-y_{P}+\text { height }-y_{Q}}{2}
\end{aligned}
$$

\section{Energy-saving lamp tail defect check}

In energy-saving lamp post check, some tail defects can be detected. But there are other defects that cannot be found, such as the cases shown in Fig. 4, tail inside and outside energy-saving lamp post. Tail is tungsten wires that could not be truncated in tail region. For the case of filament inside energy-saving lamp post, we need to check that inside area of lamp post if not null and then judge as tail flaw.

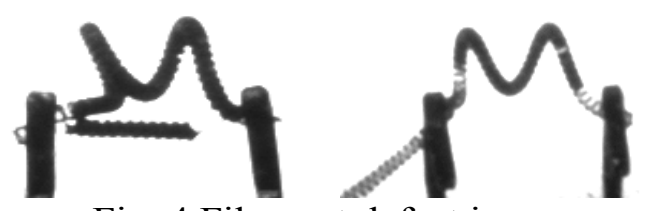

Fig. 4 Filament defect image

In Eq. 3 and Eq. 6, the height $h_{P Q}$ and width $d_{n}$ of energy-saving lamp post have been given. After test in industrial environment, rectangle check area is selected, between two posts of energy-saving lamp, under the height of energy-saving lamp post, as shown in Fig. 5. Point $M$ is a vertex of the rectangle. The $\mathrm{x}$-axis and $\mathrm{y}$-axis of point $\mathrm{M}, x_{M}$ and $y_{M}$, is given in Eq. 7. The length and width of this rectangle is given in Eq. 8. We then can get the rectangle data and check if filament here and then tail flaw.

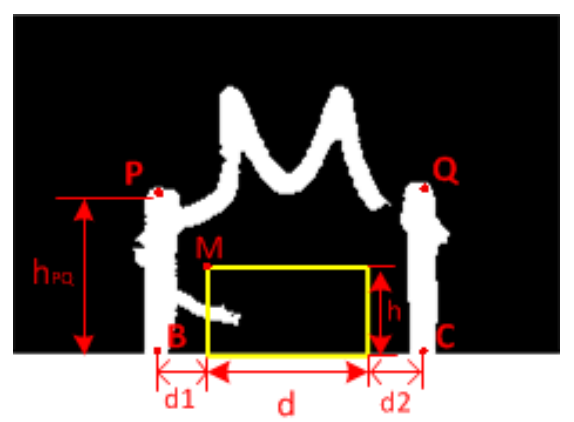

Fig. 5 Image of filament inside energy-saving lamp post

$$
\left\{\begin{array}{l}
x_{M}=x_{B}+2.5 \times d_{n} \\
y_{M}=\text { height }-\left(h_{P Q}-4 \times d_{n}\right)
\end{array}\right.
$$




$$
\left\{\begin{array}{l}
h=h_{P Q}-4 \times d_{n} \\
d=x_{C}-2.5 \times d_{n}-x_{M}
\end{array}\right.
$$

\section{Energy-saving lamp filament defect check}

Apart from post and tail flaw, there may be some flaws in energy-saving lamp filament. The flaws of energy-saving lamp tungsten are mainly expressed as clustering and twin wire. For the case of such flaw, we can figure out the proportional value of lamp tungsten and judge as clustering and twin wire if the proportional value more than the threshold.

Firstly, we select ROI area, between B and C point and two times distance of $d_{n}$ contracted respectively, the height of filament as ROI area height, point $\mathrm{K}$ as a vertex of ROI rectangle area, as shown in Fig. 6. The $\mathrm{x}$-axis and $\mathrm{y}$-axis of point $\mathrm{K}, x_{K}$ and $y_{K}$, is given in Eq. 9. The height and width of ROI area is figured out in Eq. 10, then we get ROI area.

$$
\begin{aligned}
& \left\{\begin{array}{l}
x_{K}=x_{B}+2 \times d_{n} \\
y_{K}=0
\end{array}\right. \\
& \left\{\begin{array}{l}
d_{R O I}=x_{c}-2 \times d_{n}-x_{K} \\
h_{R O I}=\text { height }
\end{array}\right.
\end{aligned}
$$

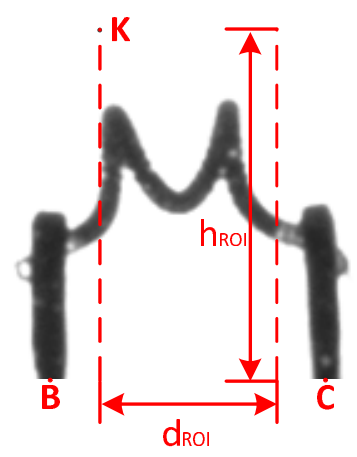

Fig. 6 ROI area

Secondly, we need to select a good tungsten powder coating image, and figure out the proportional value between tungsten pixels and total pixels in ROI area, that as template parameter. As shown in Eq. $11, S_{R O I}$ is the tungsten pixels in ROI area, $S_{\text {all }}$ is total pixels in ROI area, and $k_{R O I}$ as the proportional value.

$$
k_{\text {ROI }}=\frac{S_{R O I}}{S_{\text {all }}}
$$

In practical application, the corresponding proportional value $\mathrm{k}$ should be worked out in the same manner. Then we compare $k$ and $k_{R O I}$ value. As shown in Eq. 12, if $\mathrm{k}$ equal or greater than the sum of $\mu$ and $k_{R O I}$, the result $\mathrm{P}$ is 0 , that means bad image. $\mu$ is float parameter here, defined in $3 \%$ actually.

$$
P=\left\{\begin{array}{l}
0, k \geq \mu+k_{R O I} \\
1, k<\mu+k_{R O I}
\end{array}\right.
$$

Over the method mentioned above, we can distinguish out tungsten image with flaw better. Fig. 7 shows clustering and twin wire image and their separate binary image. 


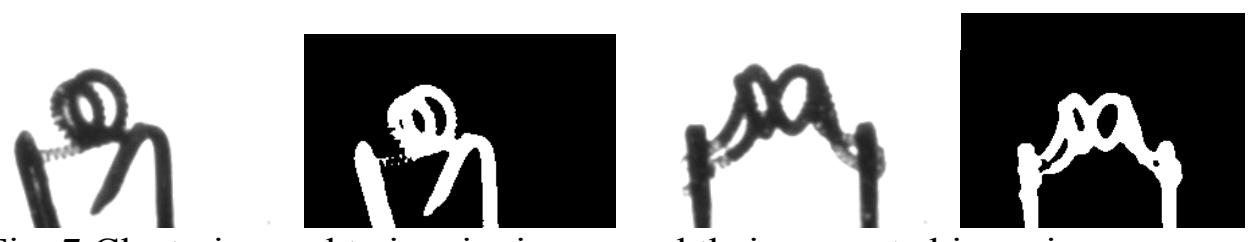

Fig. 7 Clustering and twin wire image and their separate binary image

\section{Experiment and Conclusion}

In actual production energy-saving lamp stays 1200 milliseconds in each line station. After performance test of image processing algorithm repeatedly, it processes image and outputs the result in 120 milliseconds, so it can satisfy real-time requirement of collection and processing in industrial production. We collect 12 group test data, and each group checks 500 images of tungsten filament. As shown in Table 1, the information such as the number of unqualified filament, the number of no filament, is given. The effective percentage and accuracy percentage in Table 1 is worked out from Eq. 13 and Eq. 14 respectively.

Table 1. Sample check list

\begin{tabular}{|c|c|c|c|c|c|}
\hline & $\begin{array}{c}\text { test } \\
\text { number }\end{array}$ & $\begin{array}{c}\text { number of } \\
\text { unqualified }\end{array}$ & $\begin{array}{c}\text { number of no } \\
\text { filament }\end{array}$ & $\begin{array}{c}\text { effective } \\
\text { percentage }\end{array}$ & $\begin{array}{c}\text { accuracy } \\
\text { percentage }\end{array}$ \\
\hline 1 & 500 & 8 & 32 & $92.00 \%$ & $98.29 \%$ \\
\hline 2 & 500 & 11 & 48 & $88.20 \%$ & $97.57 \%$ \\
\hline 3 & 500 & 12 & 85 & $80.60 \%$ & $97.11 \%$ \\
\hline 4 & 500 & 7 & 49 & $88.80 \%$ & $98.45 \%$ \\
\hline 5 & 500 & 8 & 46 & $89.20 \%$ & $98.24 \%$ \\
\hline 6 & 500 & 8 & 25 & $93.40 \%$ & $98.32 \%$ \\
\hline 7 & 500 & 10 & 50 & $88.00 \%$ & $97.78 \%$ \\
\hline 8 & 500 & 7 & 11 & $96.40 \%$ & $98.57 \%$ \\
\hline 9 & 500 & 9 & 81 & $82.00 \%$ & $97.85 \%$ \\
\hline 10 & 500 & 13 & 62 & $85.00 \%$ & $97.03 \%$ \\
\hline 11 & 500 & 16 & 32 & $90.40 \%$ & $96.58 \%$ \\
\hline 12 & 500 & 14 & 66 & $84.00 \%$ & $96.77 \%$ \\
\hline sum & 6000 & 123 & 587 & $88.17 \%$ & $97.73 \%$ \\
\hline
\end{tabular}

effecttive percentage $=\frac{\text { sum }-(\text { number of unqualified }+ \text { number of no filament })}{\text { sum }}$

$$
\text { accuracy percentage }=\frac{\text { sum }-(\text { number of unqualified }+ \text { number of no filament })}{\text { sum }- \text { number of no filament }}
$$

The algorithm proposed in in this paper can distinguish the deficient energy-saving lamp with lamp post, tail and filament flaw and enhance the quality of product efficiently. The experimental result proves the algorithm good stability and the worth of application.

\section{Acknowledgement}

The work is supported by Zhejiang Provincial Natural Science Foundation of China (Grant No. LZ14F030001, LY12F01022). 


\section{References}

[1] Luo Jing, Dong Tingting, Song Dan, Xiu Chunbo. A Review on Surface Defect Detection, Journal of Frontiers of Computer Science and Technology.2014,8(09): 1041-1048.

[2] Kumar Ajay. Computer-vision-based fabric defect detection: a survey. IEEE Transactions on Industrial Electronics.2008,55(1): 348-363.

[3] Malamas, E. N., et al. A survey on industrial vision systems, applications and tools. Image and vision computing.2003,21(2): 171-188.

[4] Sun Chenggang. Technique and Facilities of Rotating Phosphor Coating for Spiral Compact Fluorescent Lamps. CHINA LIGHT \& LIGHTING. 2010,(11):28-31.

[5] Luo Jing,Lin Shuzhong,Ni Jianyun,Song Limei. Battery surface quality detection using variation model and morphology. Journal of Computational Information Systems.2010,6(4):1351-1356.

[6] Zhang Peng,Wang Runsheng. A Survey of Detecting Regions of Interest in a Static Image. Journal of Image and Graphs.2005,10(2):142-148.

[7] Yao Chunlian,Guo Keyou, Jiang Huming. Design of Embedded Electronic Video Processing System Based on DM6467. Advances in Mechanical and Electronic Engineering. Springer 2013: 487-492.

[8] Pawate, B. I. Developing embedded software using DaVinci OMAP technology. Synthesis Lectures on Digital Circuits and Systems.2009,21: 1-152. 